\title{
EPISTEMOLOGIA DE LUDWIK FLECK PRESENTE NA PROPOSTA DE MENDES SOBRE REDES DE ATENÇÃO À SAÚDE
}

\section{EPISTEMOLOGY BY LUDWIK FLECK PRESENTED IN MENDES PROPOSAL ON HEALTH CARE NETWORKS}

\author{
Rodrigo Juliano Grignet ${ }^{1}$ \\ Adriana Zilly ${ }^{2}$ \\ Reinaldo Antônio Silva-Sobrinho ${ }^{3}$
}

\begin{abstract}
Resumo: A pesquisa analisou na obra Mendiana a presença de reflexões que remontem o pensamento Fleckiano, presente nas redes de atenção à saúde. Para tanto utilizou-se um estudo revisional narrativo e toda prospecção textual foi retirada da obra do autor, observando expressões ou estruturas que reforcem, apontem ou apresentem indicativos do conteúdo epistemológico de Fleck. Elaborou-se um quadro sinóptico com alinhamento ao pensamento do pesquisador e verificou-se que a estrutura de pensamento percebida na ideia de construção das redes de atenção à saúde são reflexos de modificação contínua destas redes. Portanto, a circulação e modificação de conceitos e paradigmas nos grupos de coletivo de pensamento estão sutilmente alinhadas com o pensamento do epistemólogo. Trata-se de ideias circulantes e determinantes, observadas contundentemente na necessidade de transferência contínua de conhecimento, delegando responsabilidade e autonomia aos partícipes das redes.
\end{abstract}

Palavras-chave: Fleck; Epistemologia; Redes de Atenção à Saúde; Coletivo de Pensamento; Sociedade.

Abstract: The research analyzed in the work Mendiana the presence of reflections that go back the Fleckiano thought, present in the health care networks. For this purpose a narrative review was used and all textual exploration was taken from the author's work, observing expressions or structures that reinforce, point or present indicative of the epistemological content of Fleck. A synoptic framework was drawn up in line with the researcher's thinking and it was verified that the structure of thought perceived in the idea of the construction of health care networks is a reflection of the continuous modification of these networks. Therefore, the circulation and modification of concepts and paradigms in the collective groups of thought are subtly aligned with the thinking of the epistemologist. These are circulating and determining ideas, strongly observed in the need for continuous transfer of knowledge, delegating responsibility and autonomy to network participants.

Keywords: Fleck; Epistemology; Health Care Networks; Collective Thought; Society.

\footnotetext{
${ }^{1}$ Mestre em Ensino pela Universidade Estadual do Oeste do Paraná (UNIOESTE). Docente do Curso de Medicina da Universidade Federal da Integração Latino-Americana (UNILA), Foz do Iguaçu, Paraná, Brasil. E-mail: rodrigo.grignet@unila.edu.br

${ }^{2}$ Doutora em Ciências Biológicas pela Universidade Estadual de Maringá (UEM). Docente do curso de Enfermagem, do Programa de Pós-Graduação (Mestrado) em Ensino e do Programa de Pós-Graduação (Mestrado) em Saúde Pública em Região de Fronteira da Universidade Estadual do Oeste do Paraná (UNIOESTE), Foz do Iguaçu, Paraná, Brasil. E-mail: aazilly@ hotmail.com

${ }^{3}$ Doutor em Ciências da Saúde pela Universidade de São Paulo e Pós-Doutorado em Saúde Pública pela Universidade de São Paulo (USP). Coordenador do Programa de Pós-Graduação Stricto Sensu em Saúde Pública em Região de Fronteira. Laboratório de Epidemiologia e Pesquisas Operacionais em Saúde LEO da Universidade Estadual do Oeste do Paraná (UNIOESTE), Foz do Iguaçu, Paraná, Brasil. Email: reisobrinho@unioeste.br
} 


\section{Introdução}

\subsection{A Epistemologia de Fleck como ponto de observação inicial}

O desenvolvimento de uma rede de saúde adequada às necessidades da comunidade pressupõe contínua vigilância dos profissionais envolvidos nos processos construtivos em todos os níveis de atenção, desde a gestão do trabalho, e até mesmo, o entendimento contextual de como a doença interfere no indivíduo, no grupo e no seu ambiente.

Admitir que o conhecimento continuamente produzido pelas redes de atenção derivado das experimentações, contato e inter-relação dos círculos de pensamento (usuários, gestores e profissionais), não geram produtos na construção do conhecimento é ingênuo. A prática da atenção em saúde neste momento, pode ser observada no contexto dos fenômenos históricos e sociais, de sua validação por estruturas políticas, no sentido das relações humanas e não por critérios definitivos, logo, com impacto na dinâmica das inteirações entre e intergrupos.

É sob esse entendimento que Fleck (1986) colabora na compreensão e percepção constitutiva do caráter histórico e social dos conceitos da ciência envolvidos nos processos de trabalho em saúde, de tal modo que possa explicitar o vínculo entre a ciência produzida em saúde, com o pensamento estruturado presente nas redes de atenção à saúde (RAS) propostas pelos defensores do Sistema Único de Saúde (SUS).

\subsection{Breve histórico de vida do epistemólogo Ludwik Fleck}

Ludwik Fleck (1896-1961) foi um médico de origem polonesa com raízes na tradição judaica. Clínico atuante e pesquisador voltado para a área da bacteriologia, microbiologia e imunologia. Abordou também estudos em sociologia, filosofia e história da ciência. Sua obra é reconhecida como uma das pioneiras da abordagem sociológica no estudo do conhecimento científico (LORENZETTI; MUENCHEN; SLONGO, 2013; FERREIRA, 2012).

Seu caminho foi tortuoso, o que explica a pouca exposição de suas obras. Ele e sua família foram vítimas da ocupação nazista na Polônia durante a Segunda grande Guerra, enviados para os campos de concentração de Auschwitz e Buchenwald (SCHÄFER; SCHNELLE, 2010; CURI; SANTOS, 2011). 
O epistemólogo e seu núcleo familiar superaram os tempos de guerra, diferentemente de alguns amigos, colegas e outros familiares. Mesmo durante o período de guerra, prosseguiu com seus estudos e pesquisa, desenvolvendo uma nova técnica de preparação da vacina contra o tifo a partir da urina dos portadores, o que despertou grande interesse das forças alemãs, motivo pelo qual teve sua vida preservada (SCHÄFER; SCHNELLE, 2010; MASSONI; MOREIRA, 2015). Os autores relatam também, que ao voltar à Polônia, no pós-guerra, dedicou-se à docência universitária, tornando-se membro de importantes grupos de pesquisas científicas de seu país. Entre 1946 e 1957, dedicouse a intensa atividade acadêmica, orientou quase cinquenta teses de doutorado, publicou 87 artigos e participou de um número expressivo de eventos científicos, um deles inclusive no Brasil.

A sua intensa participação na produção de conhecimento relacionado aos diversos aspectos da saúde lança um olhar sobre o comportamento dos grupos de pensadores, que de alguma forma interagem para a formação dos coletivos de pensamento, o que é observável nas redes de atenção que compõem o SUS.

\subsection{A visão do epistemólogo Ludwik Fleck sobre o pensamento coletivo}

Fleck descreve de maneira incisiva a importância do padrão de pensamento apoiado no coletivo. Afirma que "a forma de pensar é definida como um perceber dirigido com a necessária elaboração intelectual e objetiva do que é percebido" (FLECK, 1986, p.145). Desse modo, a maneira de pensar proposta caracteriza questões que interessam ao coletivo e que consideram como factível, proporcionando também um viés coercitivo sobre os indivíduos do grupo definindo o que não pode ser pensado por estes.

Assim, o coletivo de pensamento está presente sempre que duas ou mais pessoas comungam o mesmo estilo de pensamento. $\mathrm{O}$ coletivo consiste numa comunidade com foco em determinado estilo de pensar, que não é, nem deve ser confundida com uma comunidade fixa, como uma classe social ou profissional única, uma vez que um mesmo indivíduo pode participar de mais de um coletivo de pensamento e, em mais de um estilo de pensar (FLECK, 1986; FERREIRA, 2012).

Os principais fatores envolvidos no processo cognitivo, o indivíduo, o coletivo e a realidade objetiva (aquilo que deve ser conhecido), precisariam também ser observados, pois as inter-relações entre os mesmos existem. O coletivo de pensamento é a soma de indivíduos produtores de conhecimento baseada na circulação de ideias. O indivíduo 
dentro do coletivo raramente estaria plenamente consciente do estilo de pensamento presente em seu grupo, pois este, quase sempre exerce uma força absoluta e indelével sobre o seu pensamento minimizando ou abolindo outras formas de pensamento (MASSONI; MOREIRA, 2015).

Dentro da estrutura geral do coletivo de pensamento, o epistemólogo identifica a formação de dois círculos formados pelos integrantes desse coletivo: há um pequeno círculo esotérico, formado por uma minoria de maior domínio intelectual no campo de conhecimento em questão, envolvido por um grande círculo exotérico, formado pelos indivíduos não especialistas na área. A partir do saber especializado (esotérico) surge o saber exotérico caracterizado pela simplificação da ciência nas publicações de divulgação popular. Um indivíduo poderia pertencer simultaneamente a diversos coletivos de pensamento, transitando livremente entre eles, garantindo a circulação inter e intracoletiva de ideias (FLECK, 1986; MASSONI; MOREIRA, 2015).

A epistemologia do pensador está presente em referências de pesquisa no Brasil, sendo utilizada como marco teórico para estudos na grande área da saúde e nas pesquisas de ensino em ciências reafirmando a relação epistemológica com a percepção Fleckiana da saúde (PFUETZENREITER， 2002; DELIZOICOV， 2004; DELIZOICOV; CARNEIRO; DELIZOICOV, 2004; PAGLIOSA; DA ROS, 2008; PFUETZENREITER; ZYLBERSZTAJN, 2008; SILVA-ARIOLI et al., 2013; SCHVEITZER et al., 2013).

Em outro estudo, o epistemólogo é utilizado para categorizar a área de ensino de ciências, e se organiza em coletivos de pensamento que buscam problematizar a forma de disseminação sistemática de conhecimentos científicos. O autor aborda como estes coletivos estabelecem a comunicação em vários níveis percebidos, permitindo a constituição da pesquisa em ensino de ciências no Brasil como um campo social de produção de novos conceitos científicos (DELIZOICOV, 2004).

Nas observações propostas pelo pensador, todas as afecções que atingem o ser humano e seu entorno pertencem a uma complexidade ímpar, com diversos elementos que a permeiam, mas não é um produto por si, simplista. Ao se definir cientificamente os contornos da doença propriamente dita, classifica-se também um doente e se produz uma necessária prática social médica. A doença, por si mesma, não pode ser explicada em uma totalidade, seria um fenômeno que envolve um todo que não pode ser reduzido a partes segmentadas e simplificadas (LÖWY, 1994). Parte-se do pressuposto que a produção do conhecimento ocorre de bases teóricas e metodológicas compartilhadas por coletivos de pesquisadores (JUNGHANS, 2011). 
Um forte exemplo é dado na obra de Fleck (1986), ao considerar equivocada a afirmação de Schaudinn ao definir a Spirochaeta pallida como causadora da doença sifilítica (FERREIRA, 2012). Para o pensador, a sífilis abordada desta forma é desvinculada de um coletivo de pensamento e por consequência um estilo de pensamento não existiria neste contexto, pois esta referência é dada pelo grupo de indivíduos que de alguma forma, tem contato ou lidam com a doença em questão. Outro pesquisador teria identificado protozoários como causadores da sífilis (SOUZA, 2005).

Neste caso, o epistemólogo foi direto ao dizer que o conhecimento sobre sífilis tinha que ser investigado como qualquer outro sucesso da história das ideias, como um resultado do desenvolvimento e da coincidência de algumas linhas coletivas de pensamento. Se sua descoberta fosse acolhida de forma adequada e recebido divulgação pertinente no meio do coletivo de seus pares, o entendimento da sífilis, poderia ser conceitualmente outro (FERREIRA, 2012).

De acordo com a percepção atual, alguns tipos de sífilis seriam considerados como relacionados com outras doenças causadas por corpos inclusos; em outros casos, seriam consideradas como sintomas de outras doenças (MASSONI; MOREIRA, 2015). O conceito Fleckiano permeia a noção que o ambiente social que se constrói o conhecimento define sua importância perante o grupo que o definiu.

Ao sugerir um programa de epistemologia comparada, para ser estudado por filósofos, historiadores, sociólogos, antropólogos e linguistas (LÖWY, 1994), o epistemólogo deseja destacar a forma complexa da estrutura dos fatos científicos, indicar que esta mesma constituição ocorre por contingências dinâmicas e flexíveis, que concatenam aos fatores conjuntos no processo do conhecimento.

Assim, a realidade objetiva (o que deve ser conhecido), é sempre um elemento que se reorganiza tanto a partir da contribuição de ideias preestabelecidas e de fortes tradições transmitidas. Este processo de realimentação histórica, nunca esgota, é muito possível e provável "que não existam erros completos nem tampouco verdades completas" (FLECK, 2010, p. 61).

$\mathrm{Na}$ análise de Massoni e Moreira (2015) a estrutura das redes sociais com sua divisão do trabalho, cooperação, troca mútua de ideias, controvérsias, hierarquizações e conflitos, estariam presentes em todos os aspectos das atividades científicas, corroborando o pensamento do epistemólogo. Assim, um coletivo bem organizado englobaria uma quantidade de conhecimento muitíssimo superior à capacidade de qualquer indivíduo. Ressaltando, que qualquer tipo de aprendizado está ligado a alguma 
tradição social, e que palavras e costumes já seriam suficientes para formar um vínculo de pensamento coletivo.

Dessa forma, pretende-se analisar os aspectos que ressaltam e marcam o estilo de pensamento presente em textos constitutivos da área que defendam a implantação das RAS no serviço público brasileiro (uma análise das redes a luz da epistemologia Fleckiana). E de forma mais objetiva, explicitar as relações entre a estrutura de pensamento dos profissionais da saúde e os conceitos de circulação de ideias presente na construção contínua das redes de atenção; além de apresentar as implicações destes aspectos para a pesquisa em ensino de saúde e seus desafios.

\title{
2 Desenho Metodológico
}

Trata-se de um estudo revisional narrativo das referências, pois não utiliza critérios explícitos e sistemáticos para busca e posterior análise crítica da literatura se comumente utilizados no campo educacional, na área da saúde são denominados como revisão narrativa (ELIAS et al., 2012), por permitir estabelecer relações com produções anteriores, identificando temáticas recorrentes, apontando novas perspectivas, consolidando uma área de conhecimento e constituindo estrutura para orientações de práticas pedagógicas voltadas aos parâmetros de formação de profissionais para atuarem na área (ROTHER, 2007).

Borba e das Neves (2015) afirmam que nesse tipo de estudo, são analisadas as produções bibliográficas em determinada área, fornecendo informações de um tópico específico, evidenciando ideias, métodos, subtemas que têm recebido maior ou menor ênfase na literatura selecionada. Cordeiro et al., (2007) complementam a afirmativa e acrescentam que:

\begin{abstract}
A revisão da literatura narrativa ou tradicional apresenta uma temática mais aberta; dificilmente parte de uma questão específica bem definida, não exigindo um protocolo rígido para sua confecção; a busca das fontes não é prédeterminada e específica, sendo frequentemente menos abrangente. A seleção dos artigos é arbitrária, provendo o autor de informações sujeitas a viés de seleção, com grande interferência da percepção subjetiva (CORDEIRO et al., 2007, p. 429).
\end{abstract}

A seleção dos textos inseridos na obra de Mendes obedeceu à lógica do alinhamento com a epistemologia Fleckiana, ao buscar inferências ou estruturas de pensamento que se direcionem ao proposto por Mendes (2011) na construção contínua das Redes de Atenção à Saúde do SUS (GROOPMAN, 2001; GAWANDE, 2002; CAMPOS, 2003; SINGH, 2008; MENDES, 2011; MASSONI; MOREIRA, 2015). 
A perspectiva desta pesquisa foi observar estes aspectos estruturais, que ao estarem presente na escrita de Mendes e desenvolvidas em seus textos reforcem ou ressaltem o pensamento do epistemólogo. A extração dos textos na obra Mendiana permitiu traçar linha comparativa de alinhamento com a estrutura propositiva do pensador fleckiano.

Para adequada visualização e análise da estrutura de pensamento presente, que respondam aos questionamentos iniciais, foi elaborado um quadro sinóptico com a exposição dos textos referenciados e a seleção destes ocorreu com leitura voltada à prospecção semântica que indicasse a relação com o que é exposto na obra epistemológica.

Os textos foram citados de forma direta, sem alterações, permitindo a aproximação e reflexão necessária ao observar o estilo de pensamento que permeia Mendes ao utilizar-se da obra de Fleck.

A escrita estruturada e presente na obra de Mendes que contribuem para esta pesquisa foi alinhada aos descritores utilizados para busca no referencial da ideia de pensamento coletivo proposto por Fleck, onde a circulação intracoletiva de ideias é responsável pela formação dos pares que compartilham o estilo de pensamento, círculo esotérico. A circulação intercoletiva de ideias é a responsável pela disseminação, popularização e vulgarização dos estilos de pensamento para outros coletivos - círculo exotérico (DELIZOICOV, 2004).

$\mathrm{Na}$ etapa final da análise textual traçou-se um paralelo entre os autores para evidenciar o estilo de pensamento em foco, novamente ressaltando que o objetivo era buscar a existência de estruturas semânticas que atestassem a relação entre os autores e suas obras (Figura 1). 
Figura 1: Síntese do Pensamento Intra e Intercoletivo de ideias

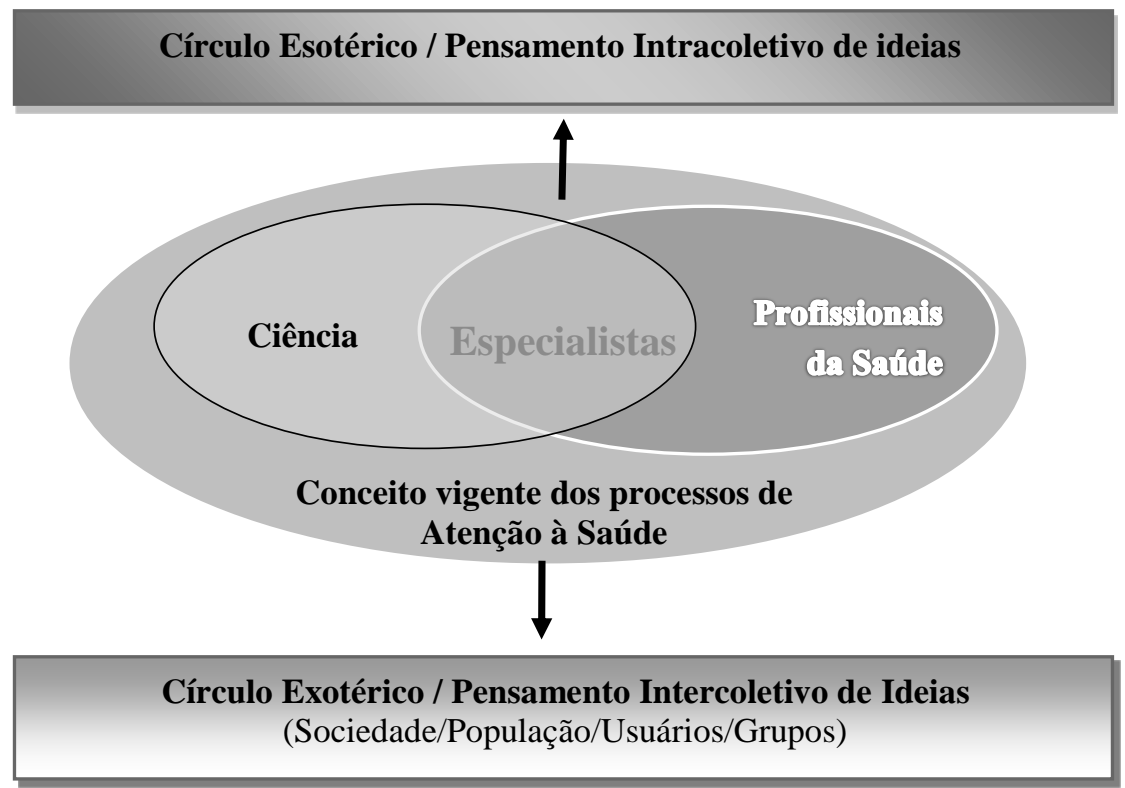

Fonte: Adaptado de Nascimento (2005)

\section{Resultados}

Os resultados obtidos podem ser visualizados no quadro 1 , que identificam autores inseridos na obra de Mendes ou ele próprio, em trechos expositivos, que ao serem selecionados evidenciam a correlação da estrutura textual alinhado ao pensamento do epistemólogo. Ressalta-se que as expressões utilizadas pelos autores analisados permitem classificar seu texto em ao menos um dos conceitos fleckianos de circulação de ideias, hora reforçando o conceito de redes de saúde ao utilizar-se de outros autores pertencentes ao círculo esotérico, hora expondo suas ideias aos leitores no círculo exotérico.

Quadro 1: Relações epistemológicas existente na obra Fleckiana com alinhamento aos círculos exotérico e esotérico presentes na estrutura de pensamento Mendiano

\begin{tabular}{|c|c|c|c|c|}
\hline Autores & Trecho & $\begin{array}{l}\text { Estrutura } \\
\text { Semântica }\end{array}$ & $\begin{array}{l}\text { Círculo } \\
\text { Exo }\end{array}$ & $\begin{array}{l}\text { Círculo } \\
\text { Eso }\end{array}$ \\
\hline $\begin{array}{l}\text { Mendes } \\
2011\end{array}$ & $\begin{array}{l}\text { [...] dificuldades de rompimento de paradigmas } \\
\text { hegemônicos aceitos. }\end{array}$ & Hegemonia aceita & $\mathrm{X}$ & \\
\hline $\begin{array}{l}\text { Sing } \\
2008\end{array}$ & $\begin{array}{l}{[\ldots] \text { os sistemas de atenção à saúde são muito }} \\
\text { resistentes às mudanças. }\end{array}$ & $\begin{array}{l}\text { Resistência à } \\
\text { mudança }\end{array}$ & $X$ & \\
\hline $\begin{array}{c}\text { Linhares } \\
1999\end{array}$ & $\begin{array}{l}\text { O caráter social do conhecimento modifica-se } \\
\text { dependendo do contexto para conceitos ou noções } \\
\text { com enfoque alterado no sentido sócio cognitivo, } \\
\text { convertendo-se em símbolos de determinados } \\
\text { posicionamentos intelectuais ou políticos } \\
\text { (ideologias) [...]. }\end{array}$ & $\begin{array}{l}\text { Posicionamento } \\
\text { intelectual } \\
\text { político e } \\
\text { ideológico }\end{array}$ & & $X$ \\
\hline $\begin{array}{l}\text { Mendes } \\
2011\end{array}$ & $\begin{array}{l}\text { Sistematizar a atenção à saúde é a resposta social } \\
\text { pensada e organizada, para responder às } \\
\text { necessidades, demandas e preferências da } \\
\text { comunidade [...]. }\end{array}$ & $\begin{array}{l}\text { Demandas e } \\
\text { preferências da } \\
\text { comunidade }\end{array}$ & $\mathrm{X}$ & \\
\hline
\end{tabular}




\begin{tabular}{|c|c|c|c|c|}
\hline $\begin{array}{c}\text { Mendes } \\
2011\end{array}$ & $\begin{array}{l}{[\ldots] \text { crise contemporânea de construção do }} \\
\text { pensamento existente nos sistemas de atenção à } \\
\text { saúde }[\ldots] \text {. }\end{array}$ & $\begin{array}{l}\text { Construção do } \\
\text { pensamento }\end{array}$ & & $X$ \\
\hline $\begin{array}{c}\text { Mendes } \\
2011\end{array}$ & $\begin{array}{l}{[. . .] \text { há que se estabelecerem modificações radicais }} \\
\text { na forma que se dão os encontros clínicos entre } \\
\text { equipes e profissionais de saúde [...]. }\end{array}$ & $\begin{array}{c}\text { Estabelecer } \\
\text { mudanças na } \\
\text { forma dos } \\
\text { encontros } \\
\text { profissionais }\end{array}$ & & $X$ \\
\hline $\begin{array}{c}\text { Minayo } \\
2012\end{array}$ & $\begin{array}{l}\text { [...] a medicina social estrutura-se, com o propósito } \\
\text { de trazer para o núcleo do debate, outros } \\
\text { componentes, que não fossem apenas os de natureza } \\
\text { biomédica. }\end{array}$ & $\begin{array}{l}\text { O núcleo do } \\
\text { debate e outros } \\
\text { componentes }\end{array}$ & & X \\
\hline $\begin{array}{c}\text { Campos } \\
2003\end{array}$ & $\begin{array}{l}\text { [...] enfatiza que o trabalho das equipes e das redes } \\
\text { de saúde "deve apoiar os usuários para que ampliem } \\
\text { sua capacidade de se pensar em um contexto social } \\
\text { e cultural". }\end{array}$ & $\begin{array}{l}\text { Capacidade de } \\
\text { pensamento em } \\
\text { um contexto } \\
\text { social e cultural }\end{array}$ & $X$ & $X$ \\
\hline $\begin{array}{c}\text { Mendes } \\
2011\end{array}$ & $\begin{array}{l}\text { A atenção centrada na pessoa e na família convoca } \\
\text { habilidades comunicacionais singulares das pessoas } \\
\text { usuárias e das equipes de saúde [...]. }\end{array}$ & $\begin{array}{c}\text { Habilidades } \\
\text { comunicacionais } \\
\text { singulares }\end{array}$ & $X$ & $X$ \\
\hline $\begin{array}{c}\text { Mendes } \\
2011\end{array}$ & $\begin{array}{l}\text { Essa concepção hierárquica e piramidal deve ser } \\
\text { substituída por outra, a das redes poliárquicas de } \\
\text { atenção à saúde [...]. }\end{array}$ & $\begin{array}{c}\text { Concepção deve } \\
\text { ser substituída por } \\
\text { outra }\end{array}$ & $X$ & \\
\hline
\end{tabular}

Fonte: Mendes (2011)

\section{Discussão dos Resultados}

No pensamento fleckiano, a prática da ciência estrutura-se em dois conceitos primordiais: o estilo de pensamento e o coletivo de pensamento; além dos conceitos complementares como pré-ideia, conexão ativa, conexão passiva (FLECK, 2010).

Partindo desta observação as RAS propostas na obra de Mendes (2011) referenciam fortemente a percepção de circulação do coletivo de ideias ao afirmar em seu discurso as dificuldades de rompimento de paradigmas hegemônicos aceitos na circulação intracoletiva de ideias. Singh (2008) diz que há de se reconhecer que os sistemas de atenção à saúde são muito resistentes às mudanças, mas a situação clama por mudanças profundas e urgentes.

Assim, ao compreender o caráter social do conhecimento é possível perceber que este modifica-se dependendo do contexto, seus conceitos e noções alterando o sentido sóci-cognitivo, ao converter-se em símbolos de determinados posicionamentos intelectuais ou políticos ideologias (FERREIRA, 2012). Propõe-se que um coletivo de pensamento seria composto por diferentes indivíduos, obedecendo a certas leis comportamentais e regras psicológicas específicas, e que o coletivo de pensamento seria mais estável e consistente (FLECK, 2010).

Cabe questionar a visão de consistência nas representações sociais, sejam elas referidas à ciência ou outros momentos da vida social, tais como o cuidado em saúde. 
Porém, as expressões sociais dos coletivos de pensamento tendem a se caracterizar por sua inconsistência e contradições internas, aumentando a resistência a sua superação (MASSONI; MOREIRA, 2015).

As modificações necessárias nos modelos de atenção à saúde propostas por Mendes (2011) encontram na circulação dos coletivos a ideia e justificativa para a mudança, evidenciando as alterações que a rede pressupõe como relevante, e esse pressupor emerge da experimentação acumulada por determinado núcleo de pensamento, entendendo este sob a ótica da circulação intracoletiva de ideias.

Sistematizar a atenção à saúde é a resposta social pensada e organizada, para responder às necessidades, demandas e preferências da comunidade. Portanto, "eles devem ser concatenados pelas necessidades de saúde da população que surgem em situações demográficas e epidemiológicas especiais" (MENDES, 2011, p. 45). Fica exposto que a circulação de ideias em determinado grupo não modifica significativamente o pressuposto inicial, temos na verdade um movimento oposto, com resistência, conforme afirmado pelo referido autor.

\begin{abstract}
A introdução das redes foi feita com alguns limites: um limite ideológico por sua origem no sistema americano, um limite cultural pela tradição de fragmentação e um limite financeiro pela dificuldade de superar o pagamento por procedimentos (MENDES, 2011, p. 65).
\end{abstract}

Fleck advoga que a circulação intercoletiva de ideias só pode acontecer verdadeiramente com a transformação do conhecimento pelo grupo em que a ideia emergiu. "A circulação intercoletiva de ideias tem por derivação a mudança ou transformação dos valores dos pensamentos” (FLECK, 1986, p. 156). O autor afirma também que:

\footnotetext{
A comunicação nos coletivos, não ocorre nunca sem transformação, produzindo uma nova percepção do conhecimento circulante, de acordo com o estilo, que intracoletivamente se traduz em um reforço e intercoletivamente em uma mudança fundamental do pensamento comunicado (FLECK, 1986, p. 158).
}

As RAS percebem-se nesse movimento universal em busca da construção contínua, sustentando-se em seu movimento por caminhos coletivos que constituam uma saída para a crise contemporânea de construção do pensamento existente nos sistemas de atenção à saúde. Há claras evidências, em vários países, que a organização sistemática reforça a circulação de ideias no intercoletivo, melhora substancialmente os indicadores sanitários, epidemiológicos e econômicos dos sistemas de saúde proposto (MENDES, 2010). 
Existe forte relação entre o pensamento estrutural Fleckiano na construção do coletivo e a formatação de ideias que corroborem a adoção de caminhos inovadores para o exercício efetivo em redes de atenção à saúde, reproduzindo de forma subjetiva o modelo de circulação de ideias e a transformação dos modelos assistenciais postos a comunidade.

Baseado na epistemologia de Fleck $(1986,2010)$ e no conceito de circulação intra e intercoletiva de ideias de Delizoicov (2004) percebe-se a evolução estrutural da abordagem dada à saúde no Brasil e no mundo, e seus componentes conceituais que convergem por esta circulação de ideias. Mendes (2011) refere-se a esta mudança como uma nova forma de gestão do processo de trabalho em redes. Ele atesta que:

Essa concepção hierárquica e piramidal deve ser substituída por outra, a das redes poliárquicas de atenção à saúde, em que, respeitando-se as diferenças nas densidades tecnológicas, rompem-se as relações verticalizadas, conformandose redes policêntricas horizontais (MENDES, 2011, p.84).

A afirmação do autor corrobora com a concepção Fleckiana que as ideias circulantes modificam a percepção do grupo em que se apresenta, na verdade, o referencial epistemológico permite ver que sem uma circulação de ideia apropriada à transformação, deforma a proposta inicial, inviabilizando-a. Citando:

O fracasso dos sistemas de atenção à saúde fragmentados, no plano micro, determinou a falência da principal instituição que o sustentou, a consulta médica de curta duração. Por isso, para se ter sucesso no manejo das condições crônicas, há que se estabelecerem modificações radicais na forma que se dão os encontros clínicos entre equipes e profissionais de saúde. Não é demais afirmar que uma atenção adequada às condições crônicas institui uma nova clínica (MENDES, 2011, p. 293).

Ao afirmar que é necessária uma nova clínica, Mendes (2011) evidencia novamente que a circulação de ideias no intercoletivo, propostas na epistemologia de Fleck, é o veículo necessário para vencer barreiras e mudar os paradigmas assistências nas redes de atenção; equivalente às demais afirmativas evidenciadas.

Para Gawande (2002) orientar-se somente pela doença não capacita a lida com o sentir-se mal, com os sofrimentos não causados por enfermidades com agentes definidos. Essa condição prevalece no cotidiano clínico. Presume-se que metade das pessoas atendidas pelos médicos da Atenção Primária em Saúde - APS apresenta sintomas físicos não explicáveis clinicamente. Há, contudo, evidência importante de que os sintomas físicos medicamente não explicáveis podem ser abordados com efetividade por terapia cognitiva (GROOPMAN, 2001). 
Quando toda a biomedicina não é mais capaz de dar respostas rápidas e dirimir os casos de endemias e epidemias, a medicina social estrutura-se, com o propósito de trazer para o núcleo do debate, outros componentes, que não fossem apenas os de natureza biomédica. Em que um dos determinantes incluídos na discussão, foi à educação (MINAYO, 2012).

Campos (2003) enfatiza que o trabalho das equipes e das redes de saúde deve apoiar os usuários para que ampliem sua capacidade de se pensar em um contexto social e cultural. Para o autor, isto poderia ser realizado tanto durante as práticas clínicas quanto as de saúde coletiva. Ratifica também que "caberia repensar modelos de atenção que reforçassem a educação em saúde, objetivando com isso ampliar a autonomia e a capacidade de ação interventiva das pessoas sobre suas próprias vidas” (CAMPOS, 2003, p. 9).

O epistemólogo apresenta-se fortemente evidenciado nas relações do círculo exotérico e esotérico quando da proposição da apropriação do conhecimento pelo primeiro círculo. Desse modo, o círculo exotérico não interfere de forma direta com aquela criação dialógica do pensamento, mas sim indiretamente, através da ponderação do círculo esotérico, vinculando os membros do coletivo de pensamento com criação de elementos produzidos pelo estilo de pensamento e aceitáveis através da confiança intergrupos (CONDÉ, 2016).

Existe ainda a possibilidade que no círculo esotérico ocorra à formulação de ideias, ao que Fleck (1986), chama de circulação intracoletiva, produzindo formatos de pensamento responsável pela veiculação de saberes científicos, a partir da distinção entre a ciência propriamente dita saber especializado - círculo esotérico, e a ciência social do saber popular círculo exotérico (OLIVEIRA, 2016). Esta perspectiva fica evidente na obra de Mendes (2011) quando afirma que:
A atenção centrada na pessoa e na família convoca habilidades comunicacionais singulares das pessoas usuárias e das equipes de saúde. De parte das pessoas usuárias implica a estruturação do processo narrativo, a reflexão sobre sua condição de vida, a reconstrução de sua história e a identificação com a saúde; em relação as equipes de saúde, o desenvolvimento da escuta e das habilidades comunicativas, a busca da empatia para com o outro e a reflexão sobre a prática mediada pelo saber individual e pela vivência interdisciplinar (MENDES, 2011, p. 299).

Para o autor a proposição de ideias circulantes e determinantes, observadas contundentemente na necessidade de transferência contínua de conhecimento, delegando assim responsabilidade e autonomia aos partícipes das redes. 


\section{Considerações finais}

A estrutura de pensamento percebida na construção das redes de atenção à saúde são reflexos reais das necessidades sociais de modificação contínua destas mesmas redes, portanto, a circulação e modificação de conceitos e paradigmas nos grupos de coletivo de pensamento estão alinhadas claramente com a estrutura do pensamento fleckiano, inclusive com proposições percebidas nos discursos sobre redes de saúde produzidas pelos autores, e elencados para essa discussão. Ao apresentar evidências da circulação coletiva de ideias e a transmissão contínua desta pelos círculos exotéricos e esotéricos, amplifica-se virtuosamente a produção de reflexões e reformulações do conhecimento primeiro.

Paulatinamente convertendo o processo de intervenção nas ações de saúde pública, fato mais organizado, compreensível e desenvolvido por grupos de coletivo que estão envolvidos na construção e aprimoramento das relações de produção dialógica. Também clarifica, e surge o encontro entre o que se está estabelecido com fator de resistência, referenciado em práticas claramente centralizadoras e hierárquicas, que revelam um estilo de pensamento controlador de uma determinada forma de pensar, contraposto ao estilo que permeia a constante necessidade de derivações e soluções, mais horizontais com redes de apoio mais capilares.

Em certo sentido, a teoria Fleckiana mostra porque a proposta da obra Mendiana ainda não se efetivou. A que se constituir coletivos mais próximos que estruturem definitivamente este coletivo ao concretizar sua hegemonia.

Este pensamento é continuamente exposto em um recorrente movimento de reconstrução e impregnação do conhecimento com saberes do coletivo que pensa e repensa a saúde. Voltando ao princípio, quando ambos os autores são confrontados como eixo desta exposição dialógica de ideias permite-se que um pensamento intracoletivo ganhe força em detrimento de outro, dentro evidentemente da complexidade dos sistemas de atenção à saúde.

Deve-se considerar a necessidade de aprofundamento da temática do eixo saúde devido a sua magnitude extrema, e os fenômenos que a envolvem são complexos, dinâmicos e interdependentes, imersos em questões que ultrapassam as fronteiras da simples assistência em saúde. Aspectos políticos, culturais, econômicos, ideológicos, regionais, climáticos e ambientais influenciam na construção de pensamento e seu 
produto final, tornando a eficiência de atenção em redes de saúde maior ou menor dependendo da soma de aspectos já elencados.

Urge a necessidade de outras pesquisas e reflexões sobre o tema com amplitude e profundidade alargadas, para uma compreensão precisa das relações epistemológicas na saúde, seus fenômenos sociais, coletivos e implicações presentes e evidenciadas no processo de formação do conceito maior do que é saúde.

\section{Referências}

BORBA, S. M. V.; DAS NEVES, R. M. C. Real primacy in the construction of scientific knowledge and the primacy of theory in the construction of scientific research: methodological contributions from Miriam Limoeiro Cardoso. Perspectiva, Florianópolis, v. 33, n. 1, p. 429 456, jan./abr. 2015.

CAMPOS, G.W.S. Paidea and a health care model: an essay on changing the ways we think and articulate for producing health care. Olho Mágico, Londrina, v. 10, n. 2, p. 7-14, abr./jun. 2003.

CONDÉ, M. L. "The reception of Ludwik Fleck in Brazil: from an anonymous visitor to a renowned thinker". Transversal: International Journal for the Historiography of Science, Belo Horizonte, v. 1, n. 1, p. 46-51, dez. 2016.

CORDEIRO, A. M. et al. Systematic review: a narrative review. Revista Colégio Brasileiro de Cirurgiões, Rio de Janeiro, v. 34, n. 6, p. 428-431, nov./dez. 2007.

CURI, L. M.; SANTOS, R. C. Ludwik Fleck and the sociocultural analysis of science(s). Hist. Ciên. Saúde-Manguinhos, Rio de Janeiro, v. 18, n. 4, p. 1169-1173, out./dez. 2011.

DELIZOICOV, D. Research in science teaching as applied human sciences. Cad. Bras. Ens. Fís, Florianópolis, v. 21, n. 2, p. 145-175, ago. 2004.

DELIZOICOV, N. C.; CARNEIRO, M. H. S.; DELIZOICOV, D. The motion of blood in human body: from the knowledge production context to its teaching. Ciência educação, Bauru. v. 10, n. 3, p. 443-460, set./dez. 2004.

ELIAS, C. S. R. et al. Whenistheend? A narrative review on the termination of the school term for mentally disabled students. Revista Eletrônica Saúde Mental Álcool e Drogas SMAD, Rio Claro, v. 8, n. 1, p. 48-53, jan./abr. 2012.

FERREIRA, M. T. Review1. Genesis and development of a scientific fact. Plural, São Paulo, v. 19, n. 2, p. 165-170, 2012.

FLECK, L. La génesis y El desarollo de un hecho científico. 1. ed. Madrid: Alianza Editorial, 1986.

FLECK, L. Gênese e Desenvolvimento de um Fato Científico. Tradução de Georg Otte e Mariana Camilo de Oliveira. 1. ed. Belo Horizonte: Faberfactum, 2010.

GAWANDE, A. Complications: a surgeon's notes on an imperfect sciene. 1. ed. New York: Metropolitan Books of Henry Holt and Company, 2002. 
GROOPMAN, J. Second opinions: eight clinical dramas of decision making on the front lines of medicine. New York: Penguin, 2001.

JUNGHANS, M. Translating Fleck: an interview with Georg Otte and Mariana Camilo de Oliveira. História. Ciência Saúde-Manguinhos, Rio de Janeiro, v. 18, n. 4, p. 1151-1158. out./dez. 2011.

LORENZETTI, L.; MUENCHEN, C.; SLONGO, I. I. P. The reception of the epistemology of fleck for the research in science education. Ensaio Pesquisa em Educação em Ciências, Belo Horizonte, v.15, n. 3, p. 181-197, set./dez. 2013.

LÖWY, I. Ludwik Fleck and the history of science today. Hist.cienc.saude-Manguinhos, Rio de Janeiro, v. 1, n. 1, p.7-18, jul./out. 1994.

MASSONI, N. T.; MOREIRA, M. A. Fleck's Epistemology: A Contribution to the Debate on the Nature of Science. Alexandria: Revista de Educação em Ciência e Tecnologia, Rio Grande do Sul, v. 8, n. 1, p. 237-264, maio. 2015.

MENDES, E. V. As Redes de Atenção à Saúde. Organização Pan-Americana da Saúde. CONASS. 2.2011 ed. Brasília. Disponível em: <http://www.conass.org.br/bibliotecav3/pdfs/redesAtencao.pdf>. Acesso em: 20 jun. 2017.

MENDES, E. V. Health care networks. Ciência \& Saúde Coletiva, Rio de Janeiro. v. 15, n. 5, p. 2297-2305, ago. 2010.

MINAYO, M. C. S. Análise qualitativa: teoria, passos e fidedignidade. Ciênc. saúde coletiva, Rio de Janeiro, v. 17, n. 3, p. 621-626, mar. 2012.

NASCIMENTO, T. G. Contributions of Discourse Analysis and Fleck's epistemology to the comprehension of Popular Science and its introduction in science classrooms. Ens. Pesqui.

Educ. Ciênc, Belo Horizonte. v. 7, n. 2, p. 127-144, mai./ago. 2005.

OLIVEIRA, M. C. Translation collective, translation styles: On the experience of translating Ludwik Fleck in to Brazilian Portuguese. Transversal: International Journal for the Historiography of Science, Belo Horizonte, v. 1, n. 1, p. 6-11, dez. 2016.

PAGLIOSA, F. L.; DA ROS, M. A. The Flexner report: for good and for bad. Rev. bras. educ. medic., Rio de Janeiro, v. 32, n. 4, p. 492-99, out./dez. 2008.

PFUETZENREITER, M. R.A epistemologia de Ludwik Fleck como referencial para a pesquisa no ensino na área de saúde. Ciên. educ., Bauru, v. 8, n. 2, p. 147-159. 2002.

PFUETZENREITER, M.R.; ZYLBERSZTAJN, A. Perceptions of veterinary medicine students about acting in the public health area: a study based on Fleck's idea of "thought style". Ciênc. saúde coletiva, Rio de Janeiro. v.13, suppl. 2, p. 2105-2114, dez. 2008.

ROTHER, E. T. Systematic literature review X narrative review. Acta paul. enferm., São Paulo, v. 20, n. 2, p. v-vi, mar./jun. 2007.

SCHÄFFER, L.; SCHNELLE, T. Fundamentação da perspectiva sociológica de Ludwik Fleck na teoria da ciência. In: FLECK, L. Gênese e desenvolvimento de um fato científico. Belo Horizonte: Fabrefactum, s.p. 2010.

SCHVEITZER, M. C. et al. Thought styles in nursing education: scientific production in three regions of Brazil. Esc. Anna Nery, Rio de Janeiro, v. 17, n. 1, p. 60-67, jan./mar. 2013. 
SILVA-ARIOLI, I. G. et al. Promoção e Educação em saúde: uma análise epistemológica. Psicologia: Ciência e Profissão. Brasília. v. 33, n. 3, p. 672-687. 2013.

SINGH, D. How can chronic disease management programmes operate across care settings and providers? Copenhagen, Regional Office for Europe of the WHO, European Observatory on Health Systems and Policies, 2008. Disponível em:

<http://www.euro.who.int/_data/assets/pdf_file/0009/75474/E93416.pdf>. Acesso em: 17 jun. 2017.

SOUZA, E. M. A hundred years ago, the discovery of Treponema pallidum. An. Bras. Dermatol, Rio de Janeiro, v. 80, n. 5, p. 547-548, set./out. 2005.

Recebido em: 03 de novembro de 2017.

Aceito em: 20 de fevereiro de 2019. 